\title{
THICK FILM CIRCUITS: PRESENT STATE AND FUTURE DEVELOPMENT ${ }^{\dagger}$
}

\author{
J. NOVOTNY \\ ITT Components Group, Standard Elektrik Lorenz AG, Nuremberg, West Germany
}

(Received November 10, 1980; in final form May 11, 1981)

\begin{abstract}
Thick film technology has been known for about 20 years. The technique of inks, printing, firing and trimming has grown in this time to a well established state of the art. This paper gives an overview of the status today and an outlook into the future, not only for thick film, but also for hybrids built out of thick film circuits with incorporation of additional components. The paper gives the main market figures and market segments for thick film applications and mentions the main reasons for this. New fields of applications for thick film will be found in the future where considerations like reliability, space savings and cost advantage are of importance.
\end{abstract}

\section{DEFINITION}

The hybrid circuit based on thick film technology represents only one of many possible solutions for the combination of electronic components and assemblies. However, today this solution is of quite major market significance in the field of hybrid technology. We understand by this solution the combination of a planar arrangement of passive components such as conductive layers resistors, crossovers and capacitors which are applied by a screen printing process, with passive or active devices in various forms, usually in the chip form. Greatly simplified, we can describe it as very similar to pc-board technology; and since this latter technology was the starting point for the hybrid circuit, the resemblance is not surprising.

\section{MARKET SIGNIFICANCE}

In the last two years the market for thick film circuits has been the subject of considerably detailed analysis by several investigators. ${ }^{1,2,3,4}$

The results show that in 1980 the size of the world market was somewhere in the region of 2 billion dollars, of which the US market alone accounted for $70 \%$. The European market for 1979 was estimated at approximately 400 million dollars; Great Britain, France and West Germany each showed figures for 1979 of roughly 60 to 90 million dollars. The estimates

$†$ Paper originally given in the Technical Papers programme of Electronica, Munich, November, 1980. show a considerable variation because of the difficulty in ascertaining the infeed demand of several companies.

\section{MARKET SEGMENTS}

Figure 1 shows a breakdown of sales in Western Europe on the basis of the individual market segments, and highlights the major significance of telecommunications in this market. This emphasis is also reflected in the pattern of world sales. In second position we find applications in the military sphere, which is of very great importance in France and Great Britain in particular. In West Germany this segment does not have the same degree of significance. Industrial applications, the consumer and entertainments field, data processing, and automobile applications account for roughly equal shares of the market. Of considerable interest are the predicted growth rates, which show a continued bias towards telecommunications and military technology, together with above-average figures for the spheres of consumer goods and automobile technology. The market breakdown for West Germany is seen in Figure 2. Again the main emphasis is in the telecommunications field.

Predictions as to growth rates over the next 5 to 10 years range from 10 to 25 percent per year. In the field of electric components there are very few branches for which similar high figures are predicted.

These growth rates are projected for the following 
132

J. NOVOTNY

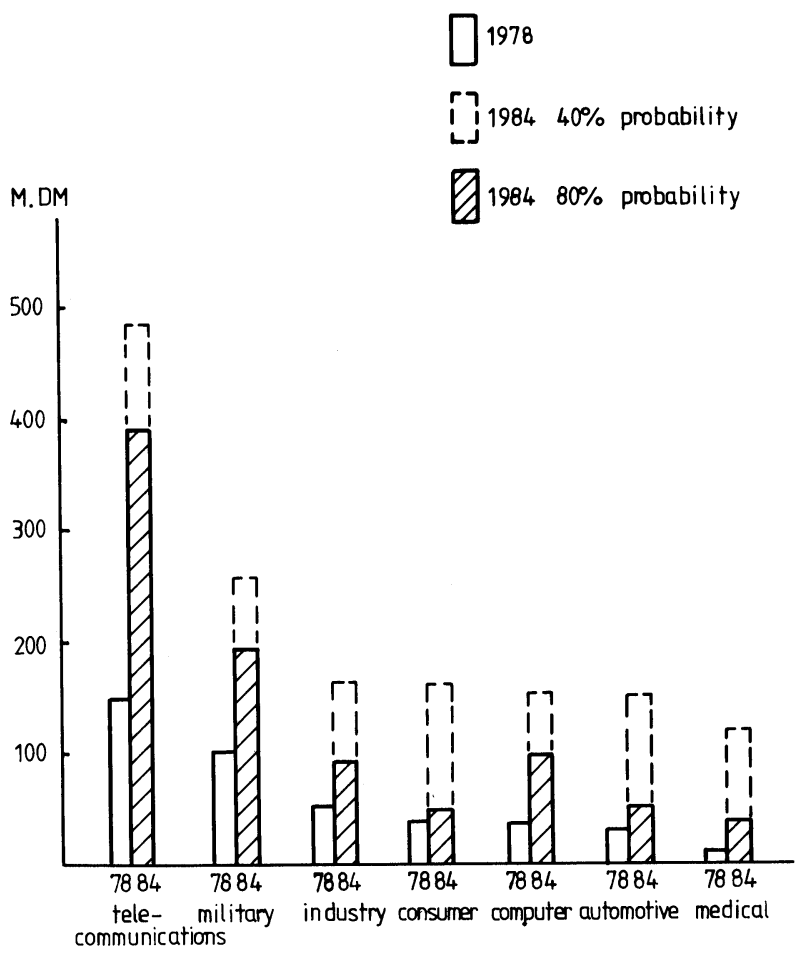

FIGURE 1 W. European Markets for Thick film - Hybrid circuits 1978 and 1984.

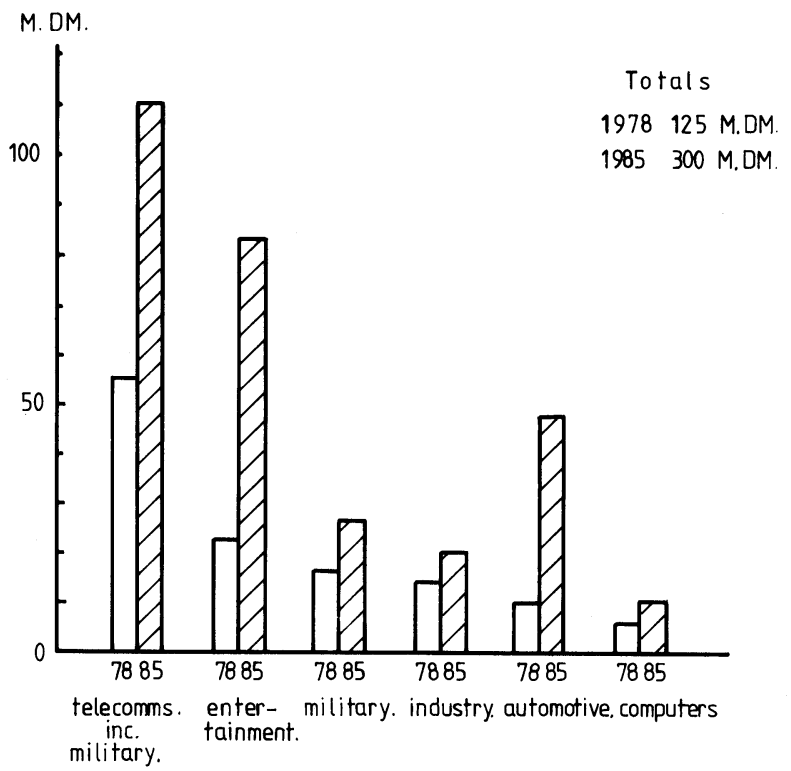

FIGURE 2 W. German Markets for Thick film - Hybrid circuits 1978 and 1985. 
reasons:

- The general expansion of the microelectronics field.

- The fact that the technological and economic characteristics and advantages of hybrid circuits are becoming more generally known, both to designers and sales staff.

- The effect of the fundamental changes becoming apparent in the telecommunications field (satellite television, digital technology, glass-fibre optical transmission).

- The availability of hybrid-compatible components.

\section{REASONS FOR THE USE OF HYBRID CIRCUITS}

The chief arguments for the use of hybrids have remained unchanged for years. These are:

- Saving of space by concentrating the circuit functions.

- High reliability as a consequence of the reduced number of connections and the improved properties of the materials employed.

- Simplified handling.

- The possibilities of reliable functional trimming.

- Cost advantages resulting from all the above factors.

\section{MATERIALS AND COMPONENTS IN HYBRID TECHNOLOGY}

\subsection{Substrates}

Alumina oxide ceramic $\left(\mathrm{Al}_{2} \mathrm{O}_{3}\right)$ has been established as the main substrate for hybrid thick film circuits for many years and will remain so in the immediate future. This material has so many merits that the disadvantage of a certain fragility can be tolerated in most applications. The enamelled steel substrates, the subject of much discussion over the last few years, without doubt have their place in certain applications; a cost benefit can be expected, for example, where particularly large areas are required, or special shapes, or particularly great mechanical stability. These factors may well become important in the automobile field. However, there are still some technical problems not finally solved, so that a broad utilisation of this substrate has not yet occurred. Steel substrates cannot be broken into smaller sections. Conventional hybrid circuits are generally produced by multiple printing; on average 3 to 5 circuits are made from a $2^{\prime \prime} \times 2^{\prime \prime}$ substrate. For this reason a certain minimum size is necessary in order to exploit cost benefits.

\subsection{Inks}

At the beginning of 1980 the dependence of price of the thick film on the precious metal market again became particularly pronounced. The conductive pastes essentially contain precious metals, silver, palladium, gold and platinum; there will be no basic change in this situation. In the immediate future, endeavours will concentrate on achieving the same properties as hitherto with a smaller proportion of precious metals. Trials with non-precious metals will certainly be carried on with vigour by all users; however, apart from one or two special developments in resistance networks, at the moment no breakthrough is in sight. Nevertheless, it is possible that in the long term several new materials will find their way into thick film technology. At present, copper seems to be the most likely candidate.

The main criteria for a good conductive path are strong adhesion to the substrate, solder leach resistance, and bondability when semiconductor chips are attached. Nowadays the resistive pastes are normally based on the bismuth-ruthenium system. The firing temperatures vary from $850^{\circ} \mathrm{C}$, for conductors and resistors to $500^{\circ} \mathrm{C}$ for overglazes. The properties of resistance layers produced in this manner are described in terms of their stability in long-term tests:

Typical stability values for tests over 10000 hours at $125^{\circ} \mathrm{C}$ are $\Delta \mathrm{R} / \mathrm{R}$ equals $\pm 0.5 \%$ for resistances between 10 ohms and 5 Mohms. (Figure 3, Figure 4) There are applications for which even narrower limits are required, and maintained. Other tests, such as resistance to moisture or rapid temperature cycling result in similar stability values. The specification for the temperature coefficient of resistors is usually $\pm 250 \mathrm{ppm} /{ }^{\circ} \mathrm{C}$ or $\pm 100 \mathrm{ppm} /{ }^{\circ} \mathrm{C}$; (Figure 5) tighter limits, such as $\pm 25 \mathrm{ppm} /{ }^{\circ} \mathrm{C}$, are required only in exceptional cases. Pastes with low TC values often have narrowed values for such data as voltage sensitivity or power loading capability.

A further improvement of resistance layers for technical reasons seems unnecessary at the moment, except for special applications (high voltage, high loading, high resistance to abrasion). Further development should be seen in terms of cost-reduction measures and fundamental changes in combination with new materials for conductive paths. 


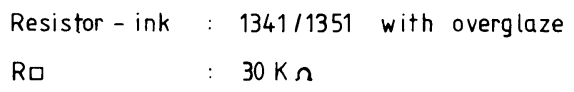

Number of resistors : 140

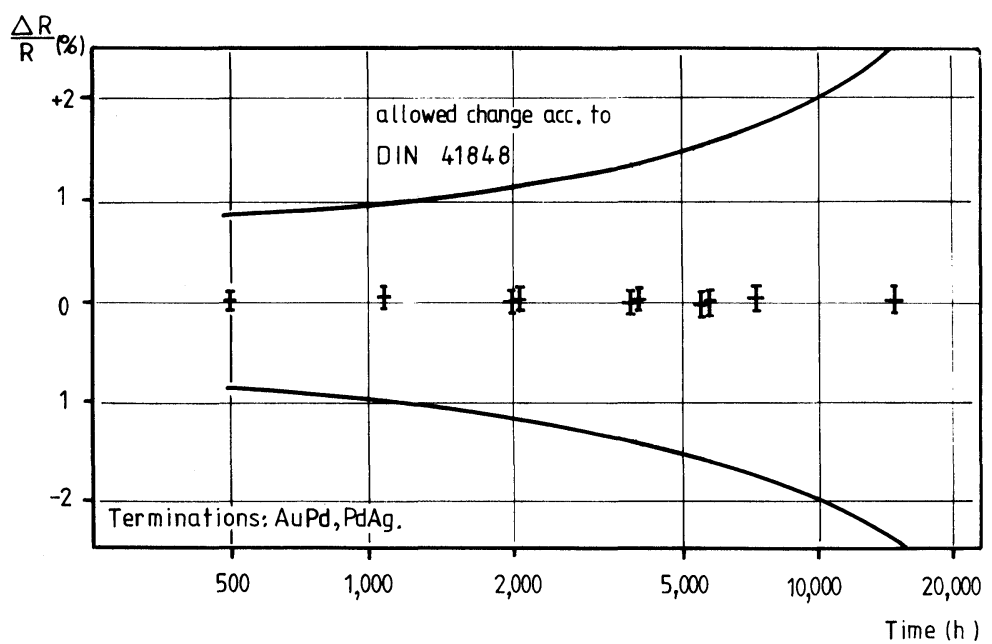

FIGURE 3 Stability of Thick film resistor at $125^{\circ} \mathrm{C}$ storage, no load $(30 \mathrm{~K} \Omega /$ sq with overglaze).
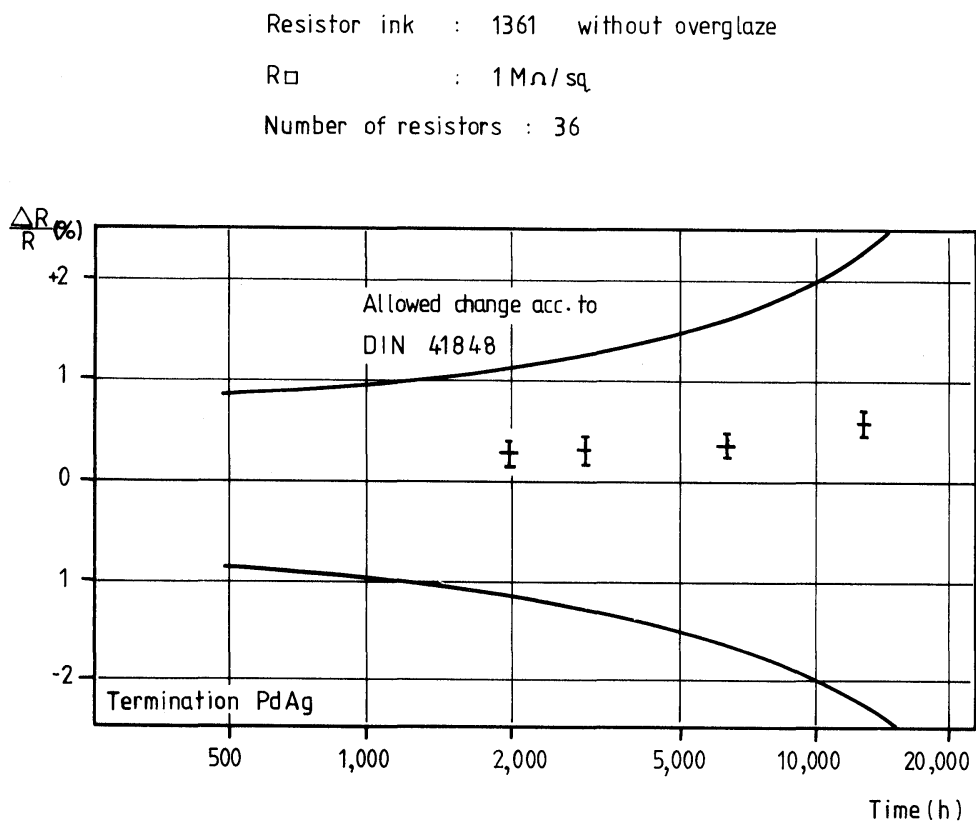

FIGURE 4 Stability of Thick Film resistors at $125^{\circ} \mathrm{C}$ storage, no lead (1M $/$ /sq without overglaze). 


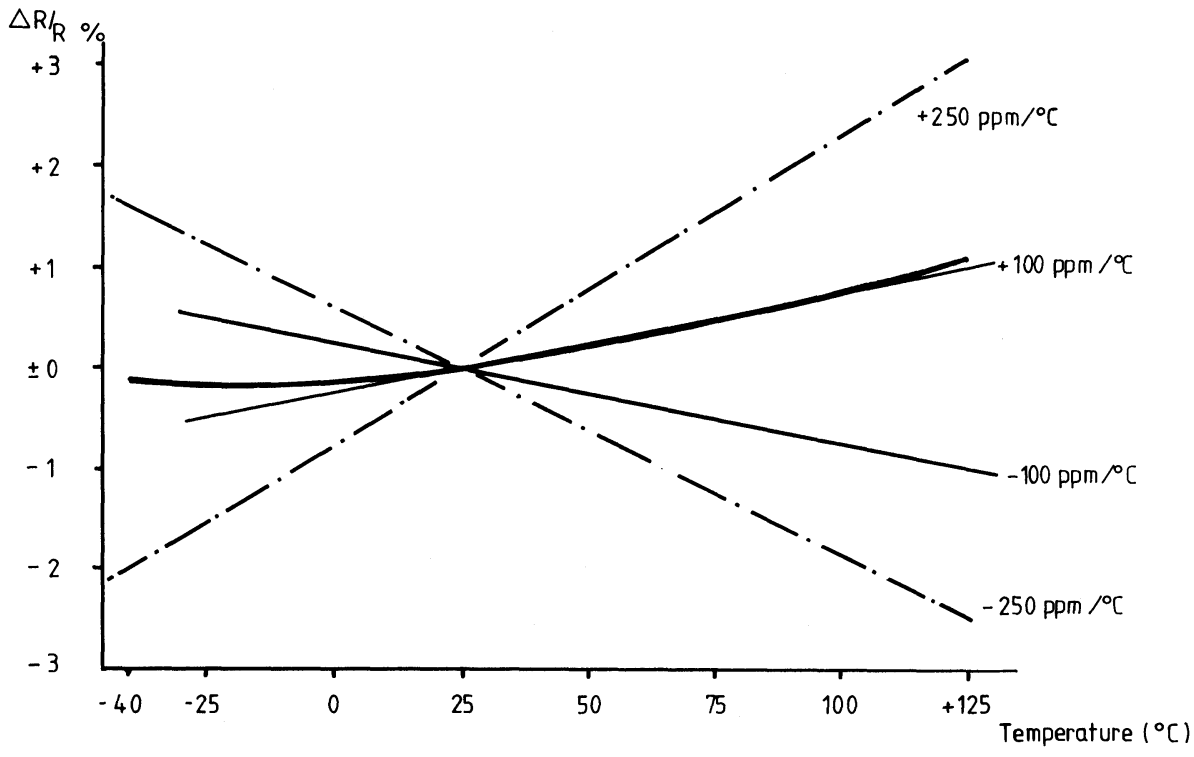

FIGURE 5 Temperature coefficient of resistance of resistors (100K/sq - 40 samples).

Dielectric pastes are of significance as glazes, as dielectric layers for crossovers, and as dielectrics for printed capacitors. With the price decrease in ceramic multilayer capacitors over the last few years, the importance of printed capacitors has not grown to the same extent as that of general film technology.

\subsection{Trimming}

It is hardly possible to conceive of a modern thick film production without the laser as a tool for trimming resistors. Since it has so far not proved possible to achieve high limits of accuracy of value after printing and firing of resistors, this instrument will continue to hold a position of major importance in the future. In fact, it is expected that the use of lasers in connection with the trimming of functions will increase. Modern laser systems offer many possibilities, either in the production of passively trimmed resistors, or when equipped with software, for production of quality control.

\subsection{Components}

Most of the components mounted on hybrid circuits are semi-conductors. The developments of these semiconductors has followed different ways in the USA and Europe, and this has led to a situation in which the processing of non-encapsulated semiconductors is much more widespread in the USA than in Europe.
Since both of these procedures offer advantages, but also have their drawbacks, work has long been underway on developing new production techniques which will combine all the advantages. From our present point of view the answers are chip-carrier technology and tape automated bonding (TAB).

The advantages in processing encapsulated semiconductors are obvious: The available components are tested, robust against handling, and do not require highly skilled personnel for their processing (mounting). The move from hand mounting towards automated operations is well underway. There is very little difference in the technology from mounting on p.c. boards, and this is the reason why many users with previous experience of p.c. board technology have introduced hybrid technology for their own requirements The necessary investments in the area of mounting are relatively low, at least as far as manual procedures are concerned.

The semiconductors employed in this technology are single transistors or diodes. If function complexity increases and integrated circuits are used, the small forms (SO-packages) are often not available, or the number of leads is too large for this form.

However, since the functional density of hybrid circuits has shown in the last few years, a similar continuous increase to that seen with integrated circuits, the need for space-saving solutions for the semiconductors is becoming more important. The ideal solution would be a space requirement not larger than 
the chip area, so that no additional space is wasted for the leads. The chip must be mounted upper side downward (towards the substrate). Although this technique is employed for some special cases, for a variety of reasons it has not become generally accepted, and it is probable that this will continue to be the case in the future.

The classical bonding technique obviously requires more space, and the time needed for the learning phase can often be rather long. On the other hand, since the semiconductor chips are usually delivered not fully tested, the reject rate is higher. In addition there are the rejects from the chip mounting and bonding operations, as well as the costs for possible rework. One solution is the chip carrier. Basically this is a new form of package, which is designed for maximum use of space and is suitable for processing by the reflow soldering technique. The assembly repackaged by this technique exhibits corresponding advantages: ease of testing and handling, and hermetic sealing. For these reasons the use of such assemblies will become of major importance when this package is fully available and when benefits become apparent as regards overall costs. However, at the moment it is often up to the manufacturer of hybrids to install the semiconductors in the chip carrier himself; and, in this case, there is no great advantage.

The utilisation of chip carriers will also become of interest for small production runs when these parts are delivered fully assembled from the manufacturer of semiconductors. On the other hand, for large runs the use of $\mathrm{TAB}$ will continue to increase. In this process, which requires relatively large investments, the semiconductors are bonded onto a film which has been laminated with copper foil and etched according to a wiring pattern (inner lead bonding). This film permits the testing of the IC as a component; in a further operation the IC with its attached leads is stamped out of the film and mounted on the substrate (outer lead bonding). This method needs approximately the same amount of space as the chip carrier, and allows extremely fast mounting of the semiconductors. For large series, and when the necessary limiting conditions are observed, this technique offers the most cost-beneficial method of mouting semiconductors. In most cases a protection for the semiconductor chips in the form of an organic covering is required, even if only as a mechanical protection for the bond connections.

\subsection{Coatings}

The most satisfactory coating of hybrid circuits represents a complex question. Depending on the application, the most varied requirements are set. Little needs to be said about hermetically sealed hybrids as their use is generally determined by the intended application or by the existing standards. Accordingly, the structural forms in this area are extremely varied.

In cases where unencapsulated semiconductors are processed, i.e. for all bonding techniques, an additional protection is absolutely indispensible, both for mechanical reasons and - because of the possible effects of moisture - on electrical grounds. The usual method is the covering of the semiconductor chips, including the bond wires, with a passivating material, generally silicone materials which are applied by dispensing units. These materials are mechanically not very robust, so that a further coating is normally required. This can be coverings of various materials such as plastic, metal or ceramic; very often, however, synthetic resin materials are used as a so-called conformal coating. It is not often that the processing methods, compositions or techniques are released for publication. The investigative methods required before quality approval are often quite protracted.

The situation is relatively simple when the semiconductors are already encased. Because of the insensitivity of the actual film elements to temperature, moisture and touch, a coating can often be dispensed with. We see here a parallel with pc boards, which are likewise seldom coated. Nevertheless protective coatings are frequently required even if only for cosmetic reasons. However, such coatings are only rarely required to withstand particularly environmental test conditions.

For this reason the use of thin dip-coatings is widespread. A coating which is too thick can entail disadvanges as a result of the differing thermal expansion coefficients of substrate, components and coating. In extreme cases this can lead to electrical failure due to cracking of soldered connections. In all probability the packaging of hybrid circuits will continue to be characterised by its present multiformity.

\subsection{Testing}

The importance of a flexible test technology cannot be overestimated. This is especially true with regard to complex circuit functions, for which hybrids are being increasingly used. A considerable expenditure of time and effort is called for in test planning, so that costs in this area can be kept under control. Admittedly, in practice it is still often the case that a special test device is produced for each product; and this certainly makes sense for simple functions or functional sequences. However, it is also necessary to make timely provisions for the testing of complex functions. In this we find 
ourselves facing the same problems which confront the manufacturers of integrated circuits. Far-sighted planning and a flexible conception of test devices are central tasks for hybrid technology in the coming years.

\section{THE SHAPE OF FUTURE TRENDS}

The previous remarks have shown that thick film technology is in a phase of diversification. In this phase the fundamental technological problems in this field can be regarded as solved, while application to a growing number of spheres constitutes the main driving force for market penetration. One or two key areas remain to be mentioned.

With the continuing growth of integration in all areas of the technology, the hybrid circuit will, more than ever, take over the task of linking together integrated circuits. Recently an American author coined the term "mechanical transformer" to characterise this function. 5 The hybrid circuit is conceived as transforming many leads on several components to a few leads on one assembly. The essential aim is less a technology for the production of thick film elements than a highly reliable interconnection technology.

Examples of this are in the increasing use of thick film multilayer circuits, for instance by several large computer firms; and the heavy emphasis on bonding technology as one which can be automated for hybrid circuits.

Two special areas of application are particularly relevant in this context: telecommunications and use in motorcars.

The telecommunications field has been preparing itself for some time for a technological revolution involving the transition from analog to digital transmission of information. This entails a major increase in the use of digital IC's requiring interconnection. This function, together with the accompanying need for high reliability and space-savings, are best served by hybrid circuits.

The application of electronics to the motorcar has long been a topic of discussion. Already there are large numbers of thick film applications in discrete electronic units; however, the main significance must be seen in the area of central electronics modules. There are already products on the market, with designations such as "on-board computer", which contain a large number of IC's to be connected to each other. In the context of further developments the significance of hybrid technology as an interconnection medium under conditions of limited space will become increasingly apparent.

Finally, it has been shown that not all system functions are amenable to digital solution. At such interfaces the development engineer is often confronted with demands for particularly tight tolerances. With its possibilities for function trimming, thick film technology is particularly suitable for overcoming such interface problems.

The future of thick film hybrid technology may be sought in all areas where a premium is placed on saving space, increasing reliability, and - last but not least - on reducing costs. And in which area is this not the case?

\section{REFERENCES}

1. Thick Film Hybrids and Networks - Western Europe, BPA (Management Services), 1979.

2. The hybrid circuit and materials market in Western Europe (Frost \& Sullivan), 1980.

3. E. Effenberger, Electrocomponent Science \& Technology, 6, p. 205, 1979.

4. D. S. Campbell \& D. Boswell, Electrocomponent Science \& Technology, 7, pp. 3-17, 1980.

5. C. T. Goddard, Proc. 29th Electronics Components Conference (Cherry Hill, N. J., May 1979), pp. 80-84, 1979. 

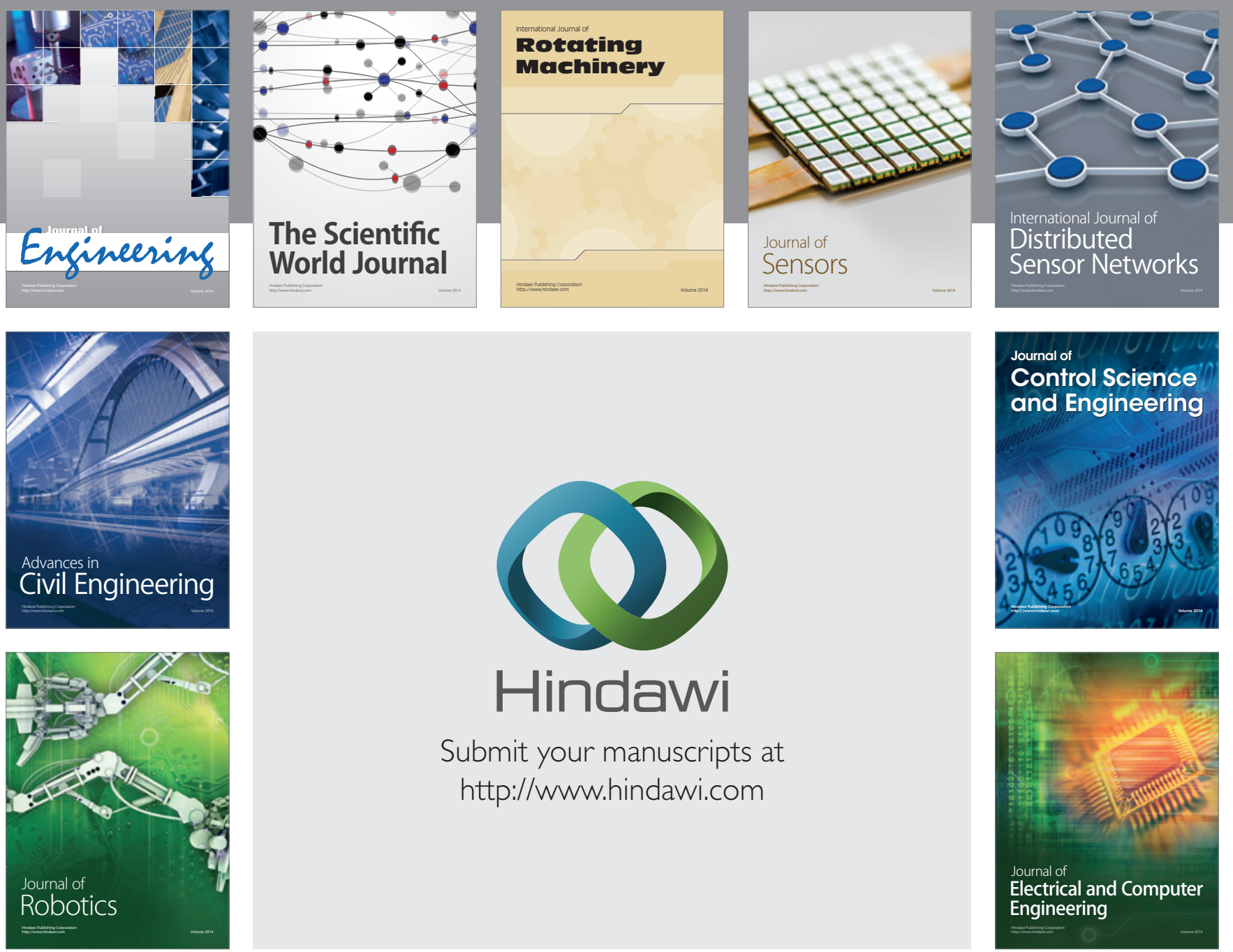

Submit your manuscripts at

http://www.hindawi.com
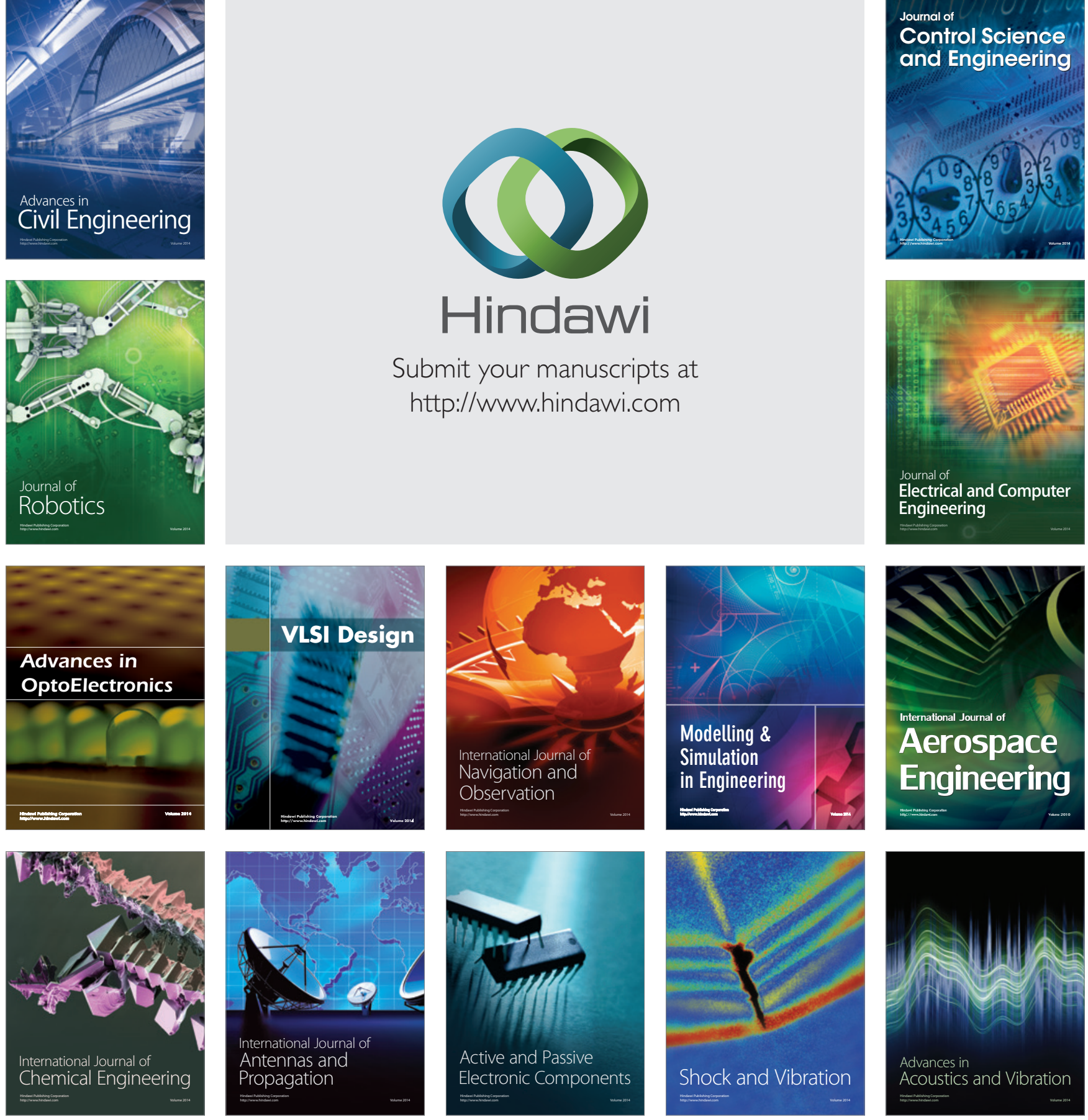